\title{
Anabases
}

ANABASES Traditions et réceptions de l'Antiquité

$23 \mid 2016$

Varia

\section{Olivier DEVILLERS \& Karin SION-JENKINS (éd.), César sous Auguste}

Hinnerk Bruhns

\section{OpenEdition}

Journals

Édition électronique

URL : http://journals.openedition.org/anabases/5693

DOI : 10.4000/anabases.5693

ISSN : 2256-9421

\section{Éditeur}

E.R.A.S.M.E.

\section{Édition imprimée}

Date de publication : 2 mai 2016

Pagination : 310-313

ISSN : 1774-4296

\section{Référence électronique}

Hinnerk Bruhns, "Olivier devillers \& Karin sion-Jenkins (éd.), César sous Auguste », Anabases [En ligne], 23 | 2016, mis en ligne le 02 mai 2016, consulté le 22 septembre 2020. URL : http://

journals.openedition.org/anabases/5693; DOI : https://doi.org/10.4000/anabases.5693

Ce document a été généré automatiquement le 22 septembre 2020.

(c) Anabases 


\title{
Olivier DEVILLERS \& Karin SION-JENKINS (éd.), César sous Auguste
}

\author{
Hinnerk Bruhns
}

\section{RÉFÉRENCE}

Olivier DEVILLERS \& Karin SION-JENKINS (éd.), César sous Auguste

Bordeaux, Ausonius, Scripta Antiqua 48, 2012, 264 p.

25 euros / ISBN 9782356130716

1 Le titre de cet ouvrage indique bien l'idée directrice du colloque dont il est issu : il s'agit de dépasser la question, mille fois posée, de la rupture ou de la continuité entre la République et le Principat, ou encore celle de l'héritage laissé par César à Auguste. Ici, la question posée est celle de " la mémoire de César, par et sous Auguste " (Roddaz, dans sa brève conclusion), question qui, on le verra, ne peut être entièrement séparée de celles de la continuité et de l'héritage.

Quinze études et une conclusion générale sont rassemblées dans cet ouvrage, distribuées sous trois grandes têtes de chapitres : I. Problématique générale et approches thématiques, II. Approches régionales, III. Approches littéraires.

La première partie couvre à peu près la moitié du livre. Elle s'ouvre, après une introduction historiographique centrée sur la France et l'Allemagne - on y reviendra à la fin - par une contribution de Martin Jehne qui compare l'organisation concrète de l'exercice du pouvoir et de la domination de César et d'Auguste : comment est réglée la disposition sur les armées, les provinces et les finances, et aussi sur les honneurs à distribuer? Du point de vue organisationnel, les solutions adoptées par César paraissent plus simples, plus directes que celles d'Auguste, mais sans différence véritable en ce qui concerne l'étendue du pouvoir de l'un et de l'autre. L'idée nouvelle que Jehne veut introduire dans le vieux débat sur César et Octavien/Auguste est celleci : ni dans l'organisation du pouvoir, ni dans la pratique du pouvoir, César n'a dépassé l'horizon du système traditionnel de patronage. Auguste, au contraire, contraint de 
gérer durablement les ressources de l'empire, a commencé à introduire des critères de décisions objectifs, initiant ainsi un processus de rationalisation administrative.

Cette réflexion sur la persistance ou non d'un horizon traditionnel de pensée est suivie par un retour sur la question de l'héritage, plus exactement sur la question de savoir ce que l'on veut dire, au fond, quand on parle d'Octave comme " héritier de César ». Paul Marius Martin reprend l'examen philologique, lexicologique et historique de l'ensemble des sources antiques qui nous renseignent sur le testament privé (!) de César et la place de l'adoption d'Octave dans ce testament. Il montre que César avait, au début, envisagé plusieurs hypothèses : naissance d'un fils légitime ; en son absence Octave hériterait des biens de César ; si César venait à mourir avant qu'un fils légitime atteigne la majorité : institution de D. Brutus comme tuteur, assisté d'Antoine et d'un conseil d'hommes de confiance ; en l'absence d'un fils légitime et au cas où Octave n'aurait pas accepté l'héritage ou serait décédé : gestion de l'héritage par Brutus, Antoine et le conseil mentionné. Puis : mention des legs au peuple. Ce n'est que peu de temps avant son départ prévu pour la guerre contre les Parthes que César aurait ajouté la clause de l'adoption d'Octave, disposition qui ensuite, dans la propagande octavienne, allait devenir la disposition centrale du testament.

Entre alors en scène " l'autre héritier » de César : Marc Antoine. Marie-Claire Ferriès évoque de façon très vivante et concrète le déroulement des premières semaines et mois après les Ides de Mars, avançant l'hypothèse que l'abolition de la dictature par un sénatus-consulte préparé par Antoine permettait de séparer la personne de César de l'institution qui avait été pervertie sous son règne. Par sa reconstruction détaillée des décisions prises au cours du printemps et de l'été 44 (lois antoniennes ou dispositions déjà prévues par César ?), l'auteur esquisse un " Antoine sous César ». Le " César sous Auguste ", cependant, a été au mieux une ombre, comme le montre Jean-Charles Balty en examinant "L'image de César dans les groupes statuaires julio-claudiens et le monnayage augustéen ». On connaît l'importance politique de ces groupes statuaires, qu'ils soient du type rétrospectif ou prospectif. Dans aucun cas, constate Jean-Charles Balty, Auguste ne se réclame de César par l'image : il n'y a pas de groupe statuaire julio-claudien qui puisse être classé de " rétrospectif ». C'est autour d'Agrippa et de ses fils que s'organisent les groupes statuaires "prospectifs " dès l'année 17. Sur les monnaies, les rappels de la figure de César disparaissent après l'an 37, fin de la première période du Triumvirat. Sous Auguste, l'encombrante paternité du dictateur n'est pas revendiquée par l'image. Elle est naturellement réclamée sous d'autres formes, que ce soit par la titulature "Divi filius ", ou par la perpétuation d'innovations dans le domaine des spectacles auxquelles Anne Bajard consacre une étude qui insiste sur le caractère de modèle, sur l'imitatio Caesaris par Auguste : spectacles de l'arène, spectacles scéniques, avec des artistes " de toutes les langues » et autres jeux et naumachies. Que la reprise de nouveaux spectacles mis en place sous César témoigne de la piété filiale d'Auguste n'est peut-être pas la principale explication qu'il faudrait mettre en avant. Si le nouveau régime reprend une telle politique de spectacles, ne devrait-on pas chercher la motivation d'abord dans la relation - politique et évergétique - entre le princeps et les spectateurs, le peuple de Rome ?

De Rome, la première contribution au chapitre des " Approches régionales " nous mène en Afrique. La continuité entre les politiques municipales de César et Auguste en Afrique étant bien connue, Stéphanie Guédon reprend le dossier de la mission conduite en 20 av. J.-C. par Cornelius Balbus chez les Garamantes, un peuple saharien qui 
occupait le Fezzan actuel, au Sud-Ouest de la Libye ${ }^{1}$. L'expédition militaire de Balbus contre les Gétules s'était poursuivie en pays garamante, devenant ainsi une expédition d'exploration des confins du monde connu qui trouvera un écho retentissant auprès des poètes. Bien entendu, la dimension politique n'était pas absente de cet intérêt de connaissance géographique : Rome montrait qu'elle pouvait atteindre les limites du monde. Cette géopolitique à la romaine, avec le projet d'une sorte de " mesure du monde ", reliait Auguste non seulement à César, mais également à Pompée.

$7 \quad$ Le cas de l'Égypte est abordé ensuite par Laurent Bricault à travers une étude du monnayage d'Auguste dans l'atelier d'Alexandrie. L'épisode César-Cléopâtre-Antoine étant terminé, la politique romaine envers l'Égypte prend un tout nouveau départ. La question "César sous Auguste " disparaît ainsi de l'horizon de cet article et cède la place à une interrogation sur le degré de romanisation des monnayages provinciaux. L'Égypte, rattachée par Auguste à l'Empire, et province atypique, apparaît du point de vue des monnaies comme l'une des provinces les plus romanisées, sans rompre avec l'héritage ptolémaïque.

8 Que la problématique évoquée dans le titre du livre se pose de façon différente pour Rome et l'Italie d'un côté, pour les provinces lointaines de l'autre, se vérifie aussi dans l'étude de François Kirbihler sur les politiques de César et d'Auguste envers l'Asie. Continuités, tout en tenant compte des contextes historiques différents, dans la réorganisation de la province et dans la politique à l'égard des cités et des populations. Continuité aussi dans la volonté de contrôler les sanctuaires. Qualifier de discontinuité le fait, pour prendre un exemple entre autres, qu'Auguste disposait en la personne de son père adoptif un divus à honorer (ou autres différences de ce genre) n'est certainement pas le meilleur choix terminologique pour aborder la question des liens entre César et Auguste. L'auteur considère d'ailleurs qu'elle est mal posée, dans la mesure où le second a vite remplacé dans la conscience des Grecs la mémoire du vainqueur de Pharsale : la revendication de l'héritage de César n'est plus à l'ordre du jour, même si tout souvenir n'a pas disparu. Question mal posée ou réponse inadaptée ? La question, me semble-t-il visait justement ce reste de souvenirs pas entièrement disparu.

Six contributions sont regroupées sous l'appellation "Approches littéraires ", abordant tour à tour des poètes, puis Tite-Live, Nicolas de Damas, Strabon, Velleius Paterculus et Tacite. Marie Ledentu analyse la présence des deux Césars et de la mémoire de la res publica chez des poètes appartenant à la même génération qu'Octave : Virgile, Horace et Properce. Ces pages témoignent à la fois de la richesse de ces sources et d'une grande finesse dans l'interprétation de ces textes poétiques conçus entre 42 et 16 av. J.-C., années de guerre civile et de bouleversements politiques. Chez ces poètes, le premier des deux Césars est décrit comme « une ombre qui traverse les textes, mais de manière suffisamment forte et complexe pour que ses contours s'y impriment dans leur ambiguïté ": l'ombre du général, du dictateur, mais aussi l'ombre (ou plutôt l'éclat ?) de l'astre signifiant sa divination.

10 Après les poètes, avec leur liberté particulière, arrivent les historiens. Bernard Mineo pose la question de savoir si le César de Tite-Live était " politiquement incorrect ", autrement dit, si l'interprétation que l'historien proposait du rôle historique de César était politiquement audacieuse au regard de la politique d'Auguste au moment où celuici prétendait rétablir la République. L'analyse des periochae (à défaut des livres perdus concernant cette période) et d'autres sources indirectes amène l'auteur à relativiser la 
thèse du pompéianisme de Tite-Live. Pour Tite-Live, la responsabilité des duces fatales pour la guerre civile impie était partagée, collective, et une telle présentation ne pouvait que contenter Auguste, qui entendait promouvoir le consensus autour de lui en rétablissant le respect du ius et du mos maiorum.

Une toute autre perspective se dégage de l'article suivant, de Guillaume Flamerie de Lachapelle, sur " César chez Nicolas de Damas : essai de lecture aristotélicienne ». Le César qui apparaît dans les parties préservées de la Vie d'Auguste est un " prototype imparfait d'Auguste ». La filiation, dans toutes ses significations, de César à Auguste est une donnée fondamentale dans cette biographie. C'est en orientant l'attention des lecteurs sur des imperfections de caractère (« le mauvais usage des passions ») et les échecs et erreurs de César en matière de vertus monarchiques (au sens aristotélicien) que l'historien peut faire apparaître Auguste, par contraste, sous un jour favorable.

L'étude suivante sur " La stasis de Pompée : Strabon et la guerre civile » aurait pu être placée dans la partie des Approches régionales. Gianpaolo Urso montre en effet que la désignation par Strabon, à première vue déconcertante, du début de la guerre civile en 49 av. J.-C. comme la " sédition » de Pompée - alors que Strabon a souvent été considéré comme philo-pompéien - exprime une vision légitimiste (à posteriori, au bénéfice du vainqueur) répandue dans la partie orientale de l'empire. L'auteur y voit une préfiguration de l'idée qui fera, plus tard, de César le premier empereur.

Isabelle Cogitore s'emploie à détecter des " reflets augustéens » dans l'image de César que nous donne Velleius Paterculus. Elle compare aux passages correspondants du Bellum Civile de César les présentations que Velleius, qui connaissait les Commentaires de César, donne dans son Histoire romaine des moments les plus importants de la guerre civile, du Rubicon jusqu'à Pharsale. Elle compare également la façon dont les deux auteurs caractérisent des personnages clés comme Caton ou Cornélius Balbus. Une perspective consciemment resserrée donc, qui réussit néanmoins à mettre en relief des différences intéressantes. Sont-elles dues davantage à la distance temporelle qui sépare Velleius de César ou bien à celle qui sépare des textes qui ne peuvent être mis sur le même plan sans toutes sortes de précautions ?

Le dernier chapitre du livre, "Permanence et transformation du modèle augustéen : Le César de Tacite ", nous mène assez loin du principat d'Auguste. Si Olivier Devillers commence par poser la question de savoir comment Tacite se situe par rapport au César de la propagande augustéenne, il termine son étude très logiquement en admettant implicitement - et même assez explicitement - qu'au tournant du ir $\mathrm{iu} \mathrm{II}^{\mathrm{e}}$ siècle ap. J.-C. la question ne se posait plus en ces termes. Ce ne sont plus les inclinaisons tyranniques du dictateur qui intéressent l'historien, mais la puissance (potentia) à la fois de César et Auguste, déterminante pour le destin de Rome. Dans sa vision de l'histoire politique, César devenait le précurseur des princes, vision qui s'imposera peu après avec les Vies des Douze Césars de Suétone.

15 Voilà donc, très sommairement résumés, les aspects essentiels traités dans ces quatorze chapitres. Ces études détaillées, brèves et concrètes du "César sous Auguste » sont précédées d'une "Introduction historiographique " rédigée par Karin Sion-Jenkis. L'auteur se concentre presqu'exclusivement sur l'historiographie allemande, depuis Theodor Mommsen. Ronald Syme est le seul historien non-allemand à être traité avec une attention particulière et à ne pas être banni dans les notes de bas de page. Karin Sion-Jenkis place son analyse de l'historiographie allemande sous les questions de la comparaison entre les personnalités et l'œuvre politique de César et d'Auguste, de 
l'héritage transmis de César à son fils adoptif, sous la question de la continuité ou de la rupture dans différents domaines de la vie publique, ainsi que sous la question des commémorations et du souvenir. N'aurait-il pas été judicieux d'y ajouter quelques éléments d'information sur les grandes tendances de l'historiographie italienne, britannique ou américaine, éléments qui auraient pu compléter la perspective développée par les treize contributions françaises et celle de Martin Jehne?

Aussi intéressante qu'elle soit, la perspective générale du volume (la concentration sur les continuités et discontinuités entre César et Auguste) mériterait d'être élargie à une interrogation sur le processus de transformation du système politique (au sens très large) qui soit moins centrée sur les deux acteurs principaux. Un tel déplacement de perspective permettrait probablement de découvrir d'autres dimensions de la question "César sous Auguste ".

\section{NOTES}

1. La carte mentionnée p. 94, n. 9 manque malheureusement dans l'ouvrage.

\section{AUTEURS}

\section{HINNERK BRUHNS}

UMR 8558 EHESS / CNRS

bruhns@msh-paris.fr 